\title{
DISTRIBUTED INFRASTRUCTURE ENABLING EFFECTIVE INTEGRATION OF EARTH OBSERVATION INFORMATION RESOURCES FOR COLLECTIVE SOLUTION OF ARCHIVING, SEARCHING, PROCESSING AND EO DATA ANALYZING TASKS
}

\author{
V. P. Savorskiy ${ }^{1 *}$, E. A. Lupyan ${ }^{2}$, V. I. Kaevitser ${ }^{1}$, M. T. Smirnov' ${ }^{1}$, D. M. Ermakov ${ }^{1}$, \\ I. V. Balashov', M. A. Bourtsev', V. Yu. Efremov', A. A. Proshin', V. A. Tolpin' , I. A. Uvarov', E. V. Flitman ${ }^{2}$ \\ ${ }^{1}$ Kotel'nikov Institute of Radioengineering and Electronics of Russian Academy of Sciences, Fryazino Department, \\ Vvedenskogo sq., 1, Fryazino, Moscow region, Russia - savor@ire.rssi.ru \\ ${ }^{2}$ Space Research Institute of Russian Academy of Sciences, 117997, 84/32 Profsoyuznaya Str, Moscow, Russia - \\ evgeny@d902.iki.rssi.ru
}

Commission IV/5

KEY WORDS: services, integration, infrastructure, distributed, system.

\begin{abstract}
:
Numerous applications of Earth observation (EO) data for Earth resource exploration both for land use management purposes and for fundamental research goals require as much as possible independent EO data sets provided for a number of data consumers, i.e. for collective (shared) data usage. In addition data distribution procedures should be accompanied with sophisticated information services enabling correct interpretation and application of provided data. As a result data providers should be able to convert data in a lot of consumer-proper formats and in some cases it should require additional data processing before data shipping. Presented work proposes basic infrastructure for collective (shared) EO data usage that enables above mentioned integration of information resources contained in typical Russian EO centers. Paper reveals architecture design, description of basic infrastructure elements, and examples of real implementations.
\end{abstract}

\section{INTRODUCTION}

Numerous applications of Earth observation (EO) data for Earth resource exploration both for land use management purposes and for fundamental research goals require as much as possible independent EO data sets provided for a number of data consumers, i.e. for collective (shared) data usage. In addition data distribution procedures should be accompanied with sophisticated information services enabling correct interpretation and application of provided data. As a result data providers should be able to convert data in a lot of consumerproper formats and in some cases it should require additional data processing before data shipping.

Main object of presented work is to design and implement basic infrastructure of collective (shared) EO data usage that enables integration of information resources contained in typical Russian EO centers. The principal approach in infrastructure development is based on data exchanges via wide use of unified integrated data sets (UIDS) which describe explored natural physical object or geographic zone, including observation data, products of data processing, metadata, browse images, and guides (glossaries, directories, descriptions, user manuals).

Basic element of developed infrastructure that should manage UIDS is Information Resource Integration Block (IRIB). IRIB should solve the following fundamental tasks in supporting distributed system functionality:

1) for each distributed system node that stores EO data IRIB should support all stack of services necessary to manage UIDS, i.e. data search and localization, data conversion, UIDS assembling, and data shipment,

2) IRIB should enable simultaneous access to several distributed system nodes which contain information describing the single natural physical object, so IRIB provides complex, i.e. multi-sources, UIDS,

3) IRIB should posses ability to transform data set to UIDS allows to build up unified interfaces of shared (collective) access for visualization of EO information resources as well for their on-line processing and analysis.

To integrate resources of distributed system along with IRIB the unified interface for collective (shared) usage (UICU) should be developed. UICU should enable the simultaneous receiving EO data and products as well their descriptive information metadata, browses, and guides. In addition, EO UICU allows to perform on-line reconfiguration of finalizing data processing by user request via special interface.

Paper describes architecture design supporting required service stack, shows details of basic infrastructure element (IRIB and UICU) functionality, and reveals results of real implementation of proposed approach in IKI GEOSMIS technology developed by Space Research Institute team.

\section{USER REQUIREMENTS FOR DISTRIBUTED EO COLLECTIVE USAGE SYSTEM (EO CUS)}

A key issue in constructing and using EO information systems is to organize the effective work of users with provided

\footnotetext{
* Corresponding author.
} 
information. To achieve this goal it is necessary to build custom interfaces that allow solving the basic problems that arise in a variety of EO systems. Naturally, every specialized monitoring system has its own special challenges, but one can outline the overall specificity, which nowadays occurs in almost all such systems. Most of modern remote monitoring systems should

1) allow to monitor large areas and quickly to provide information about monitored objects and phenomena to geographically dispersed users;

2) provide an opportunity for ongoing monitoring of controlled objects and phenomena, i.e. be designed to work with continuously updated information;

3) allow obtaining various characteristics of controlled objects and phenomena and, therefore, be focused on the use of information from various observation platform (satellites, aircrafts, in-situ etc);

4) archive data for enough long periods of observation since in many cases the objective of monitoring systems is to identify deviations from "normal" behavior of the controlled objects and phenomena;

5) be capable of working with distributed archives and databases, as the collection, analysis, processing and storage of information can take place in different geographically distributed centers;

6) due to the need of working with fairly large information flows and amounts, be mainly focused on fully automated technologies for collecting, processing, distributing and presenting the data;

7) provide users with information that has passed sufficiently deep levels of processing as well as convenient toolkits (interfaces for its analysis), and in many cases be capable of dynamic generation of certain products at the request of the user;

8) allow users to obtain and to analyze various kinds of spatial data;

9) effectively receive and integrate data from third-party monitoring systems as well as an easy access to various information products, including online services provided by the system.

\section{EO CUS ARCHITECTURE}

To work with map data EO CUS should provide at least the following functionality:

- to be able to present data on map layers;

- to own basic functions to proceed with maps;

- to be able to search catalog data and to display retrieved information;

- to be capable to can export data.

While implementing these functions in Web interfaces one should follow the next principles of their development:

- $\quad$ structural modularity;

- $\quad$ application of object data model;

- flexibility of configuration;

- easy extensibility.

As analysis reveals it is possible to split EO CUS on two functional levels which interactions are shown in Fig.1:

- Presentation level which should include Web and GIS interfaces and is based on usage of UICU;
- Application level which functionality is based on IRIB and should include:

○ Web services (including map services, metadata services, and data management services);

- System API, enabling access to data, metadata, and various resources and interfaces.

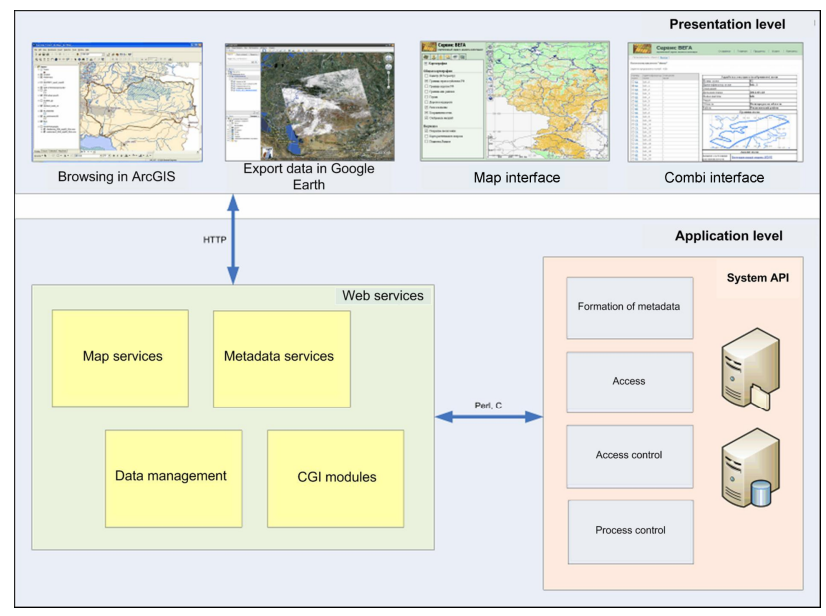

Fig.1. EO CUS architecture.

Presentation level (aka UICU) is subsystem that supports interaction with user. This level should allow user to work with system via Web interface or via specialized applications, e.g. GIS. As typical GIS is equipped with all basic tools to work with map data its interactions with EO CUS should run on data service level. This is the reason why EO CUS should be oriented on development of unified data access interfaces, i.e. UICU. UICU architecture is presented in Fig.2.

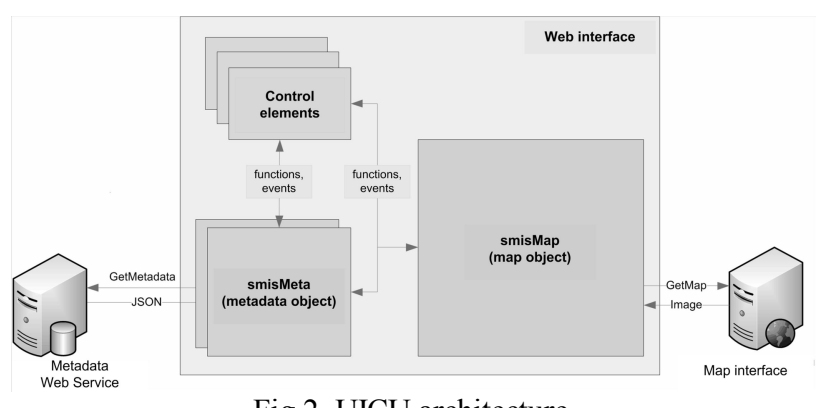

Fig.2. UICU architecture.

Work of such interfaces should be provided by kernel consisted of two object modules:

- Map module which is designed in our work as smisMap software module (see Fig.2);

- Metadata module which is is designed in our work as smisMeta (see Fig.2) software module.

Map module is advisable to implement as JavaScript object which should enable to display map information retrieved by data services. In addition, module should allow to send spatial queries and to export displaying data to print service or even to other systems.

Metadata module is advisable to implement as JavaScript object as well. This module should support catalog data search and retrieval of data necessary for information displaying on rendering service. 
Application level (aka IRIB) is subsystem that should enable interaction between interfaces and data themselves. This level must be consisted by Web services and system API (see Fig.3).

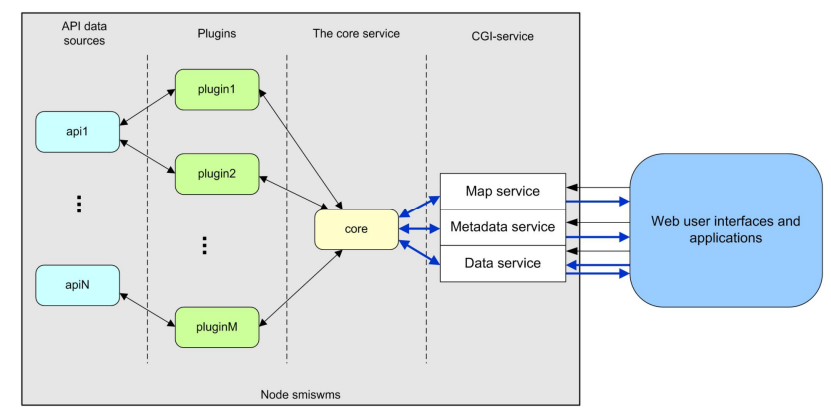

Fig.3. IRIB functional modules' structure

Web services should receive data access based on HTTP protocols. In order to provide application level functionality on application level of EO CUS three types of Web services must be included in IRIB:

- Map data Web service;

- Metadata Web service;

- Data management Web service.

Map data Web services should allow receiving map information in form of raster images. This type of services has to be based on extension of WMS standard. It should allow providing data not only for Web interfaces but as well for GIS.

Metadata Web services should allow receiving information on product availability for requested geographic region or dating interval as well for given additional criteria set. These services should be able to work with JSON or XML formats and send queries addressing any catalog in distributed data system. Returning information should contain both descriptive data and auxiliary data for map services.

Data management Web services should enable data manipulation in database via Web queries, such as adding information on contours and their attributes, their changing and deleting. Interaction with those services should be conducted in JSON format.

All three types of EO CUS Web services should be realized in frame of one common strategy i.e. should be integrated in one common service. Those common services should enables support of map data receiving (via request GetMap which should be generated in interface by smisMap module), queries on metadata receiving (via request GetMetadata which should be generated in interface by smisMeta module), requests on data addition and modification, e.g. addition of polygons and information about them. So, Web interfaces kernel should be able to provide direct interaction with metadata retrieving services and map information displaying. It is important that interactions should be possible to install with any amount of spatially distributed services.

System API is separate part of IRIB. It is the set of Perl modules, which should be located on servers and be able to get data access and to work with data on server side. All above mentioned services should work with data via those modules. Each module should take responsibility for definite data type or system part. It should allow flexible system configuration, error fixing, and fast software development.

\section{IMPLEMENTATION OF BASIC ELEMENTS}

\subsection{UICU IMPLEMENTATION}

In developing the EO CUS particular attention was drawn to the issue of designing the structure of interfaces aimed to work with data sets. Taking into account needs in reasonably clear and user-friendly interfaces which should support operations with different-type quickly updated spatial data, it was necessary to develop some general approaches to allow user easily navigate interface content. To this end the basic type of Web interface was designed, namely the UICU which structure is shown in Fig. 4.

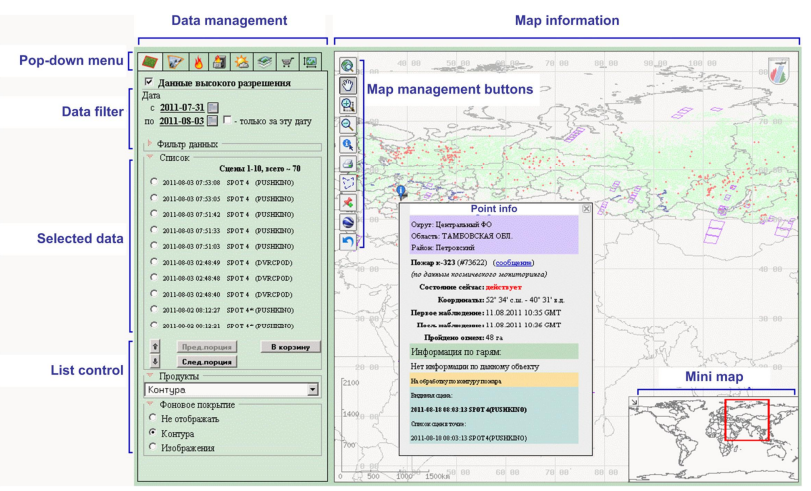

Fig.4. Basic type of UICU.

In order to support operations with different data types, UICU controls have a block structure. Each data type and typical control function matches their own module in UICU. At user level the appropriate tabs for each module and each data type are designed in UICU. This structure allows grouping various similar data and various operations on data in a convenient and clear manner. The modular structure also allows making UICU scalable, with no significant difficulty to extend the interface with new data types, groups, or additional operations. The structure allows configuring UICU to work with required types of data. It should also be noted that in variety of specialized systems which exploit the interfaces based on this principle, the processing control for the same type of the data can be performed in the tabs of the same type. It facilitates both the development of specialized interfaces and data exchange between different systems.

To work comfortably with multi-temporal datasets UICU realizes unified blocks which allow managing satellite data of different types as well as the results of their processing, e.g. working with:

- data of high spatial resolution displayed by quite localized scenes;

- data of coarse and moderate resolution with their characteristic units, "sessions", covering large areas;

- series of temporal and spatial composites of various products of satellite data processing.

There's also a special opportunity in UICU to sample different types of data layers to perform their joint analysis. Selected data layer controls are collected in a separate tab which, 
depending on the specific task of the interface instance, allows performing certain operation on that data collection.

Typical samples of tabs to manage such types of data are presented in Fig. 5.

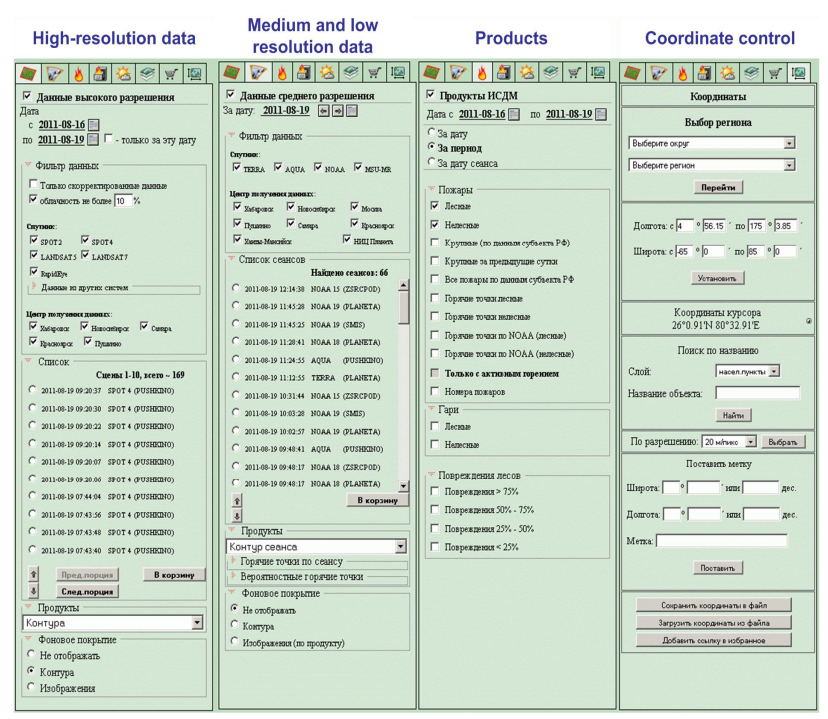

Fig.5. Typical tabs for UICU, providing data management.

UICU also provides a special opportunity to select layers of different types for their detailed joint analysis. Selected layers (scenes, sessions, etc.) are collected in a separate tab, where depending on the task of particular realization of the interface certain operations can be applied to them.

Particular attention in UICU development is paid to provision users with background implementation of obtaining data sets that are stored in distributed archives or even in different monitoring systems. So, UICU is designed to obtain data from different sources in background mode where users are not aware from which storage systems (information distribution centers) the certain data is obtained. It should also be noted that UICU is integrated with an authorization system which allows users, on authorization at the entrance to a specific interface, being able to work with a variety of distributed information resources in accordance with the policy of a particular system.

UICU also allows including a variety of functions related to data management, e.g. data inquiry from archives or sending them for specific procession, implemented in particular systems, as well as to control data export to various third-party systems.

A sufficiently rich set of control to display spatial data are supported in UICU, being almost standard for various GIS systems.

Particular attention in development of EO CUS was given to optimization of creation, development and support of UICU, implementations in particular EO information systems. Therefore the system provides various configuration modules, which allow easy controlling of basic UICU settings. Configuration modules allow flexible interface setup. Data access configuration modules contain configuration records describing sets servers for data access, parameters of WMS queries to get the data along with additional parameters such as data format. In metadata configuration modules parameters of metadata receiving as well as parameters of metadata list generation are set. smisMap configuration module allows setting both main and additional elements of map control. Overall configuration of the project is carried out in the main file of the project which lists all the configuration modules.

\subsection{IRIB IMPLEMENTATION}

One of the main tasks of EO CUS is to unify access to different kind of geospatial data. It stands for generalization of provided services and standardization of data formats. The main types of the services needed in EO CUS, like map service, map description (metadata) service and data management service, are integrated in IRIB. In frames of the presented work IRIB is implemented as a Web service smiswms which implement accesses to any kind of geospatial data in EO CUS (see Fig. 2).

The design of the service was based on OGC WMS standard (http://www.opengeospatial.org/standards/wms), extended and enhanced in our work in order to meet the requirements of existing Web interfaces and peculiarity of map generation processes. At the same time the basic format of WMS map requests, i.e. getMap, is fully supported in our work in order to ensure compatibility with third-party applications. Requests for metadata and data management were also developed taking into account the structural features of OGC standard (http://www.opengeospatial.org/standards), targeting to the practical applications. In particular, the metadata query can return the results in JSON format, supports setting the size of portions of information obtained and the work with specific filters for various data.

Thus, the smiswms interface for data provision do not depend on original formats of data storage and distribution. The smiswms service, being the SW implementation of IRIB concept, provides users access to information stored in a large number of archives and databases in different formats. Flexible support of these data sources and fast integration of new ones are ensued by the features of the service internal structure. A good example of implementation of smiswms service plug-in is a plug-in designed to work with the distributed catalog of EO data of high spatial resolution (Antonov et al, 2010). Access to these data is organized in such a way that both maps and metadata from independent catalogs of geographically dispersed centers are aggregated "on the fly", so it is insensible to the users of the service.

The smiswms service has a modular structure. The modules are grouped in fully separate specific algorithms and data access functions from general functionality, providing the work of the service as a whole. Thus, the service structure has standardized groups of modules to work with particular data - plug-ins and modules of the service core to work with plug-ins and to support external interfaces.

Software modules, necessary for support of automatic testing of services and data sources, are grouped separately. Key features of IRIB implementation as the smiswms service are: separating the processes of plug-ins and core, support of asynchronous query processing by plug-ins, and a uniform approach to exception handling. 
The core of IRIB implementation - smiswms - performs plugin execution and interaction with Web service through CGI interfaces, as well as functions necessary for service maintenance. Modules of each of plug-ins provide interface services of the smiswms core with data. Plug-ins are designed on the base of parent modules-classes and have standard interface functions for interaction with the core. This approach ensures easy adding and configuring of sets of plug-ins and comfortable joint development of the service functionality as a whole.

Yet another group of smiswms IRIB plug-ins forms testing plug-ins. This decision is defined, first of all, by specificity of algorithms of functional testing for each plug-in and, often, by the need of additional configuration of algorithm parameters for various projects. The testing plug-ins also share the unified interface for interaction with the core.

Much attention in development of the smiswms service as IRIB realization was paid to perform optimization. Optimization of services for data, metadata and maps provision was carried out in three main areas - optimization of algorithms and functions of data processing in every plug-in, capability of parallel execution of plug-ins, and application of means for caching queries. Nevertheless, in many respects better performance of the service is achieved by particular realizations implemented in design on specific data storage systems, which should be capable for fast data selection to generate maps and to provide metadata, including those from distributed sources. A successful approach in implementing an optimized storage system, which exploits the smiswms service plug-in to provide data access, is described in (Balashov et al., 2008).

The smiswms service implements IRIB using Perl interpreter and open source modules (see Fig. 6). This solution allowed using different operating environment without significant changes of the service.

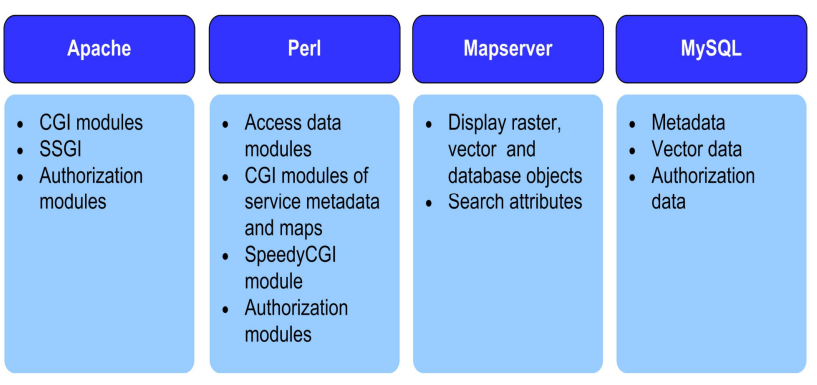

Fig.6. IRIB specific implementation

\subsection{TESTING OF THE DEVELOPED MODELS OF THE BASIC ELEMENTS IN THE EXISTING DISTRIBUTED INFORMATION SYSTEMS OF EO CUS}

EO CUS model approach in form of GEOSMIS technology (Tolpin, 2011) was tested and practiced in creating fairly complex interfaces for various monitoring systems operating in Russia. Based on this technology now established and functioning are:

- Integrated interface to work with the data of informational system of remote monitoring of the Federal Forestry
Agency of Russia (FFA ISDM) This interface allowed organizing uniform work with different types of spatial data used in the system (Bartalev et al., 2010; Efremov et al., 2011) (http://www.pushkino.aviales.ru/rus/main.sht).

- Satellite service VEGA created at the Space Research Institute of Russian Academy of Science for various tasks related to monitoring and remote assessment of vegetation status. It provides abilities to obtain and analyze more than one decade information from archives of satellite observation of Russia and the border states, in particular, LANDSAT 5 and LANDSAT 7 data. (Loupian et al., 2011a; Loupian et al., 2011b) (http://vega.smislab.ru).

- Web interface to work with data of industry-wide monitoring system of Federal Agency for Fisheries of Russia - OSM Rosrybolovstvo. This interface supports the work with both operational data about locations of vessels fishing in Russian economic zone or Russian vessels fishing anywhere in the world ocean, and with the archive information on vessels fishing since 2000. (Solodilov et al., 2011).

- Integrated data catalog data of the Scientific Center for Earth Operative Monitoring (SC EOM) of Russian Space Agency. The interface provides an opportunity to analyze data from various satellite systems. This system is focused on working with the data of Russian satellites: it contains data sets of Russian satellites Meteor-M No.1, Meteor3M, Resurs-DK, Monitor. (Bourtsev et al., 2011a) (http://thema.ntsomz.ru/geocover_v4/ntsomz.sht).

- Joint access system of the European, Siberian and Far Eastern centers for receiving and processing satellite data, SRC "Planeta". The system is designed to provide the ability to work with the results of satellite data processing obtained by leading centers of the Federal Agency for Hydrometeorology and Environmental Monitoring of Russia over all Russian territory (Bourtsev et al., 2009; Bourtsev et al., 2011b).

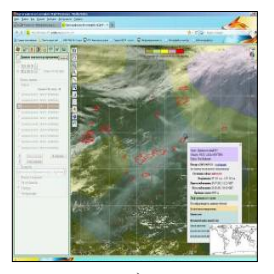

a)

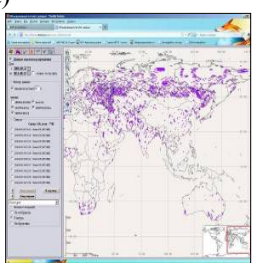

d)

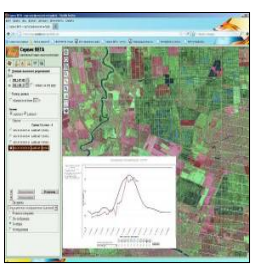

b)

e)

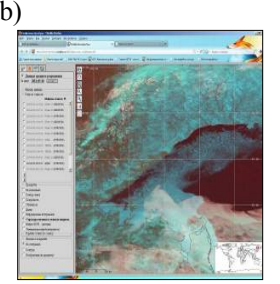

Fig. 7. Examples of interfaces of various monitoring systems based on GEOSMIS. a) FFA ISDM, b) satellite service VEGA, c) OSM Rosrybolovstvo, d) Joint catalog at SC EOM, e) Joint system of data access of SRC "Planeta" 
Examples of the interfaces, implemented in the listed systems, are shown in Fig. 7. Experience of using GEOSMIS in development of these systems has shown that technology is indeed fairly universal, easy scalable and extendable for different tasks. This makes it possible to start using the technology also in other projects conducted in the Space Research Institute and Kotel'nikov Institute of Radioengineering and Electronics.

\section{CONCLUSIONS}

1. User requirements that determine service stack of modern EO CUS should include

- $\quad$ possibilities for permanent data upgrades;

- $\quad$ support of long-term data storage;

- functionality of distributed archives and catalogues;

- functionality implementing automatic streamoriented data collecting, processing, distributing, and displaying technologies;

- tools for dynamic formation of product variety by user request;

- ability to get and analyse wide range of graphic object types;

- ability to get and to incorporate space data from different external space data systems.

2. Proposed and motivated EO CUS architecture provides basic service stack functionality. It is worthwhile to construct and develop EO CUS as two-layer model: 1) presentation layer and application layer. Presentation layer is based on map and presentation (display) modules while application one - on data integration module and application API.

3. EO CUS is implemented as IKI GEOSMIS technology. Presentation layer is implemented in GEOSMIS map data module (smisMap) and metadata module (smisMeta). Application layer is implemented in GEOSMIS as data integration module (smiswms) and applied API.

4. GEOSMIS technology formed basis of various monitoring systems based on GEOSMIS: a) FFA ISDM, b) satellite service VEGA, c) OSM Rosrybolovstvo, d) Joint catalog at SC EOM, e) Joint system of data access of SRC "Planeta".

\section{ACKNOWLEDGEMENTS}

The work was supported by Russian Foundation for Basic Research - grant No. 11-07-12028-ofi-m-2011.

\section{REFERENCES}

Antonov A.V., Bourtsev M.A, Efremov V.Yu., Kalashnikov A.V., Krasheninnikova Yu.S., Loupian E.A., Matveev A.M., Proshin A.A., Flitman E.V., 2010. Design of the Union Catalogue of Distributed Satellite Data Archives of Various Centers. Actual problems in remote sensing of the Earth from space, 7(2), pp. 84-89 (in Russian).
Balashov I.V., Bourtsev M.A., Efremov V.Yu., Loupian E.A., Proshin A.A., Tolpin V.A., 2008. Building-up the archives of results of space data processing for use in processes of dynamic formation of derivative information products. Actual problems in remote sensing of the Earth from space, 5(1), pp. 26-32 (in Russian).

Bartalev S.A., Ershov D.V., Korovin G.N., Kotelnikov R.V., Loupian E.A., Tshetinskii V.E., 2010. The main functionalities and structure of the Forest Fire Satellite Monitoring Information System of Russian Federal Forestry Agency (SMIS- Rosleskhoz). Actual problems in remote sensing of the Earth from space, 7(.2), pp. 97-105 (in Russian).

Bourtsev M.A., Voronin A.A., Eremeev V.V., Zlobin V.K., Kuznetsov A.E., Loupian E.A., Milekhin O.E., Soloviev V.I., 2009. Complex of operational processing of meteorological satellite information. Earth research from space, 1, pp. 16-23 (in Russian).

Bourtsev M.A, Efremov V.Yu., Balashov I.V., Mazurov A.A., Proshin A.A., Loupian E.A., Milekhin O.E., 2011a. Data handling system for the European, Siberian and Far Eastern centers of Roshydromet. Actual problems in remote sensing of the Earth from space, 8(3), pp. 113-119 (in Russian).

Bourtsev M.A., Emelyanov K.S., Efremov V.Yu., Loupian E.A., Mazurov A.A., Pakhomov L.A., Proshin A.A., Savorsky V.P., Artamonova E.V., 2011b. On the Possibility of Effective Interaction between Satellite Data Receiving and Processing Centers and Remote Monitoring Systems. Actual problems in remote sensing of the Earth from space, 8(4.), pp. 48-53 (in Russian).

Efremov V.Yu., Balashov I.V., Kotelnikov R.V., Loupian E.A., Mazurov A.A., Proshin A.A., Tolpin V.A., Uvarov I.A., Flitman E.V., 2011. Integrated mapping interface for operations with ISDM-Rosleskhoz data. Actual problems in remote sensing of the Earth from space, 8(3). pp. 129-139 (in Russian).

Loupian E.A., Savin I.Yu., Bartalev S.A., Tolpin V.A., Balashov I.V., Plotnikov D.E., 2011a. Satellite Service for Vegetation Monitoring VEGA. Actual problems in remote sensing of the Earth from space. 8(1), pp. 190-198 (in Russian).

Loupian E.A., Savin I.Yu., Tolpin V.A., Balashov I.V., 2011 b. Satellite Service «VEGA». E`arth from Space. 9, pp. 30-35 (in Russian).

Solodilov A.V., Pirkov V.N., 2011. Complex satellite monitoring of fishery ships. Aerospace courier, 2(74), pp. 6870 (in Russian).

Tolpin V.A., Balashov I.V., Efremov V.Yu., Loupian E.A., Proshin A.A., Uvarov I.A., Flitman E.V., 2011. The GEOSMIS System: Developing Interfaces to Operate Data in Modern Remote Monitoring Systems. Actual problems in remote sensing of the Earth from space, 8(3). pp. 93-108 (in Russian). 